ReVISTA de BIOLOGía TROPICAL

\title{
Morfoecología de gremios en escarabajos (Coleoptera: Passalidae) en un gradiente altitudinal en robledales de la Cordillera Oriental, Colombia
}

\author{
Carlos J. Moreno-Fonseca ${ }^{1} \&$ German D. Amat-García ${ }^{2}$ \\ 1. Grupo de Investigación Insectos de Colombia, Instituto de Ciencias Naturales, Universidad Nacional de Colombia, \\ sede Bogotá; cjmorenof@unal.edu.co \\ 2. Instituto de Ciencias Naturales, Universidad Nacional de Colombia, sede Bogotá. Bogotá, Colombia; \\ gdamatg@unal.edu.co
}

Recibido 24-III-2015. Corregido 20-VIII-2015. Aceptado 30-IX-2015.

\begin{abstract}
Morphoecology guild in beetles (Coleoptera: Passalidae) along an altitudinal gradient in oak forests of the Eastern Cordillera, Colombia. Bess beetles are important components on tropical forest dead wood nutrient cycling, since they act as direct consumers and ease the consumption by another organism (indirect). Studies of bess beetle ecology are scarce and have focused on communities responses to environmental changes on alimentary resources. We characterized the bess beetles guild composition in an elevation gradient, according to their differential use of resources (microhabitat) and morphological traits quantification (geometric and lineal), as a potential tool to improve our understanding on resource use and functional ecology of beetles. Three guilds (underbark, sapwood-heartwood and generalists feeders), five species and 198 familiar groups were recognized; their richness decreased as elevation increased. Changes in linear morphometric measures were influenced by elevation; morphogeometrical measures were not associated with the elevation gradient, only grouping at guild level were observed. Morphological markers analysis (morphometric) provided information to guild delimitation. Body and metatibiae shape contributed with the best information to guild grouping. Quantification of those structural markers proved the relationship between resource repartition, because they are involved in movement on wood galleries and wood consumption, this fact allowed to propose specific functional roles. The identified patterns have contributed on the understanding of functional processes in Passalidae communities and their role in ecosystem function. Rev. Biol. Trop. 64 (1): 289-303. Epub 2016 March 01.
\end{abstract}

Key words: functional ecology, guild, geometric morphometric, Passalidae.

Un gran número de estudios de carácter ecológico-funcional han contribuido al entendimiento del papel ecológico de las comunidades biológicas y de las condiciones de nicho de sus especies integrantes; al respecto, las investigaciones se han ocupado principalmente del papel de la morfología, el tamaño, las tasas metabólicas, la bioenergética y la biomecánica de los organismos para la definición de grupos funcionales y gremios (Loreau, 1998; Biewener, 2003; Blondel, 2013; Van Regenmortel, 2004; Hill, Wyse, \& Anderson, 2008).

En los Passalidae, un grupo de escarabajos saproxilófagos, se ha detectado una marcada segregación espacial dentro de los troncos que ocupan, debido en gran medida al aprovechamiento diferencial del recurso alimenticio (Schuster, 1978; Reyes-Castillo \& Halffter, 1984). Los pasálidos habitan la subcorteza, la albura, el duramen y la interfaz tronco/suelo (Reyes-Castillo, 1970; Kon \& Johki, 1987).

Los escarabajos pasálidos son insectos subsociales con una distribución pantropical (Reyes-Castillo, 1970; Mouzinho \& Da Fonseca, 1998). La totalidad de su alimentación y ciclos de vida ocurre en los troncos en descomposición (Reyes-Castillo \& Halffter, 1984), por tal motivo se ha reconocido su importante función en los procesos de degradación de madera y reciclaje de nutrientes (Castillo \& Morón, 
1992; Castillo \& Reyes-Castillo, 2003). Dicha relación ha revelado una serie de adaptaciones comportamentales y morfológicas; al respecto, todas las especies forman grupos familiares, compuestos por una pareja bisexual de adultos y su descendencia; además, los progenitores ejercen cuidado parental sobre su progenie (Reyes-Castillo \& Halffter, 1984) y elaboran nidos para huevos y cámaras de protección para pupas (Schuster, 1975; Schuster \& Schuster, 1985). Se ha comprobado la transferencia de un pool bacteriano, a través de las heces, responsable de la fermentación de la madera (Schuster \& Schuster, 1985), facilitándose así la alimentación de las larvas. Igualmente, se han descubierto importantes adaptaciones morfológicas relacionadas con la separación microzonal; estas consisten en cuerpos anchos y convexos con una menor capacidad aerodinámica, en aquellos organismos que habitan la albura-duramen y cuerpos con menor espesor y amplitud y una mayor capacidad de dispersión de vuelo en los que habitan la corteza y subcorteza (Reyes-Castillo \& Halffter, 1984; Lobo \& Castillo, 1997). Estos atributos, que pueden estar diferenciados entre las especies, además de reducir la competencia, mejoran el manejo del recurso y optimizan la habilidad de los pasálidos para desplazarse y reproducirse (Boucher, 1991; Lobo \& Castillo, 1997).

El objetivo de este estudio es la caracterización de los gremios en una comunidad de escarabajos pasálidos, mediante la cuantificación de marcadores morfológicos específicos y la ubicación microzonal de los individuos en el interior de los troncos, bajo un gradiente altitudinal en hábitats de robledal (Quercus humboldtii) de la Cordillera Oriental de Colombia. Se discuten los factores que explican la influencia de la altitud sobre la variación de tales marcadores morfológicos, basados en los gremios reconocidos.

\section{MATERIALES Y MÉTODOS}

Área de estudio: El estudio se realizó en bosques de roble colombiano (Quercus humboldtii) de la reserva Parque Natural Municipal
Robledales de Tipacoque (PNMRT), ubicada en la ladera occidental de la Cordillera Oriental de los Andes colombianos (Fig. 1). Esta área forma parte del corredor ecológico GuantivaLa Rusia-Iguaque, con bosques andinos y páramos de los departamentos de Boyacá y Santander. La reserva esta ubicada entre los 2400 y los $3400 \mathrm{~m}$. $\left(06^{\circ} 23^{\prime} 54.9^{\prime \prime} \mathrm{N}-72^{\circ}\right.$ $42^{\prime} 58.8^{\prime \prime} \mathrm{W}$ ), con una temperatura media anual de $13{ }^{\circ} \mathrm{C}$ y una humedad relativa de $78 \%$. Los muestreos se llevaron a cabo entre abril 2012 y agosto 2013.

Comunidad de pasálidos: Para determinar el gradiente altitudinal se ubicaron cuatro sitios en el interior de dos fragmentos de robledal a 2500, 2700, 3000 y 3300 m, respectivamente (Fig. 1). En cada sitio se muestrearon 16 troncos efectivos (con presencia de pasálidos) por medio de recorridos de $\approx 1 \mathrm{~km}$ lineales horizontales; adicionalmente, se efectuó la toma de datos de humedad y temperatura ambiente. Los troncos se cortaron completamente con la ayuda de hachas de 0.6 y $1.3 \mathrm{~kg}$. Se removió por completo cada uno de los estratos del tronco (corteza, subcorteza, albura, duramen e interfaz tronco-suelo), recolectando progresivamente los pasálidos asociados a cada microzona. Para el caso de los escarabajos pasálidos, además del censo de los grupos familiares, se realizó una demarcación de acuerdo a la ubicación microzonal de los individuos dentro del tronco. Debido a que se realizó un muestreo destructivo y que este se efectuó en una reserva natural, se optó por capturar solo algunos ejemplares (entre siete y diez) de adulto, larva y pupa por especie, por sitio; este procedimiento minimizó el impacto sobre la comunidad de pasálidos. Los individuos adultos capturados se preservaron en etanol (70\%), en tanto que las larvas y pupas se conservaron en una solución de acetato de etilo (50\%) para evitar el deterioro de sus tejidos blandos. Cada espécimen recolectado se identificó a nivel de especie, teniendo como referente la colección de Passalidae del Instituto de Ciencias Naturales de la Universidad Nacional 

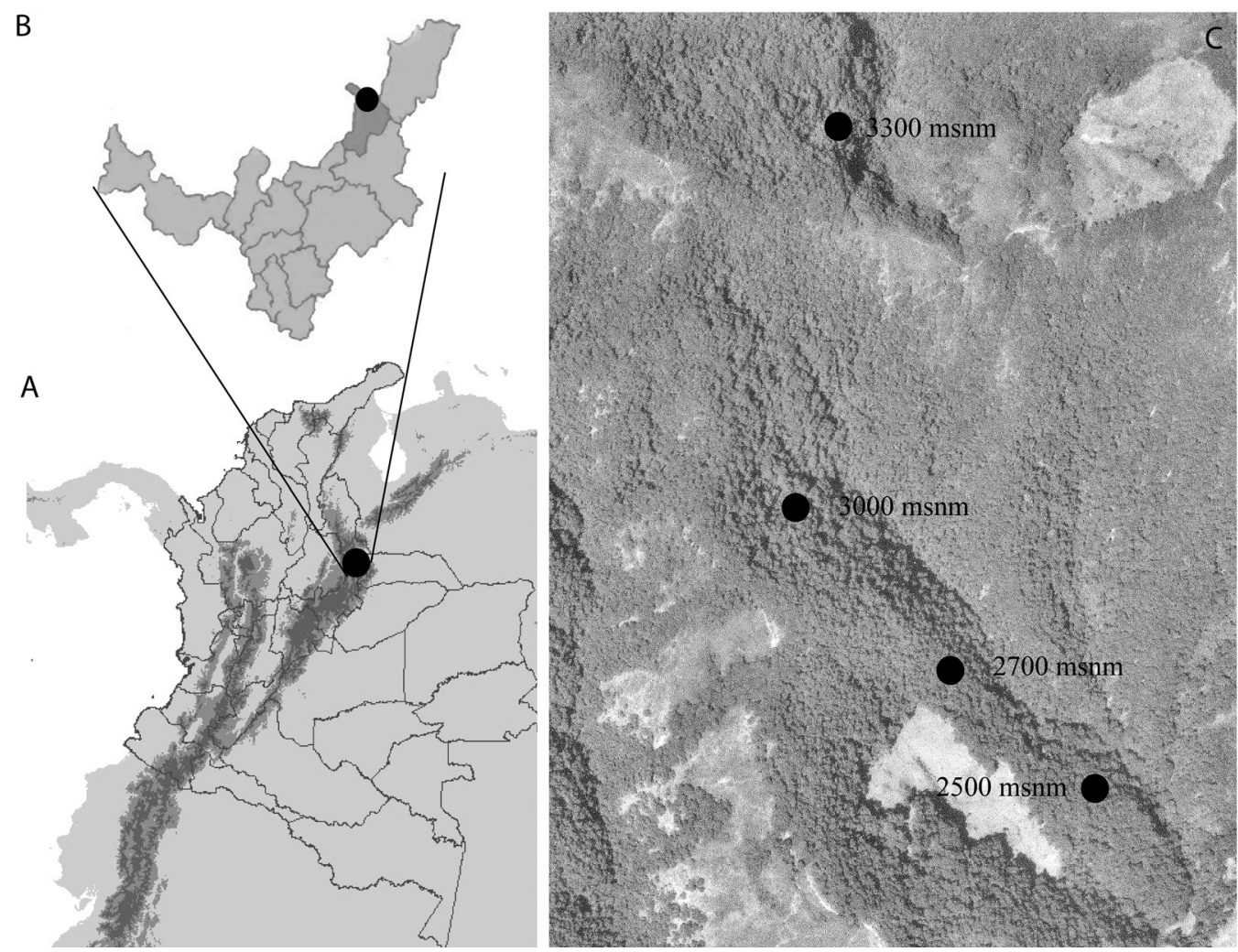

Fig. 1. (A) Ubicación nacional; (B) ubicación departamental; (C) ubicación local, con detalle de la vegetación en el gradiente altitudinal de robledal ubicado en el Parque Natural Municipal Robledales de Tipacoque, PNMRT (Cordillera Oriental, Colombia).

Fig. 1. (A) National reference; (B) regional reference; (C) Study location, detailed altitudinal points in Parque Natural Municipal Robledales de Tipacoque, PNMRT (Cordillera Oriental, Colombia).

de Colombia (ICN-UN), donde finalmente se depositó el material recolectado.

Morfometría lineal y geométrica: Para los tratamientos cuantitativos lineales se tuvieron en cuenta las variables adoptadas por Lobo y Castillo (1997) como la longitud total (L), el ancho de los élitros (W), el grosor corporal (H) y su peso fresco (BW). La toma de datos se efectuó con la ayuda de un calibrador digital y una balanza digital de precisión marca Radwag modelo PS450X. El análisis morfogeométrico se realizó con base en la forma general del cuerpo, las alas y las protibias. Se analizaron entre siete y diez especímenes por especie/gremio correspondientes a cada punto altitudinal. Cada uno se digitalizó con la ayuda de un estereoscopio y de una cámara de alta resolución; las imágenes se tomaron con una resolución de 2000 x 1000 pixeles, y se estandarizaron de forma tal que la posición, aumento y tamaño del objeto no afectara los resultados. Para la descripción corporal se digitalizaron los especímenes en los planos dorsal y lateral, en el caso de alas anteriores y tibias solo se trabajó el plano dorsal. Estas estructuras fueron extraídas de cada espécimen, con la ayuda de un equipo de microdisección.

La descripción de los dos primeros marcadores morfológicos (cuerpo y alas) se realizó utilizando puntos homólogos o marcadores de reconocimiento morfológico, ubicados en un plano de dos dimensiones (landmarks); estos se seleccionaron según los criterios de Bookstein 
(1991). Bajo estos principios, para la descripción general del cuerpo, se utilizó un total de 19 puntos homólogos dorsalmente y 17 lateralmente, de los 36 puntos totales, 13 compartieron su ubicación en ambos planos (Fig. 2); dichos puntos de orientación actuaron como referentes tridimensionales al combinar ambos planos (Rauum, 2006). Por su parte, la ubicación de puntos homólogos en las alas se realizó siguiendo los patrones de venación (Villemant, Simbolotti, \& Kenis, 2007; Klingenberg, Debat, \& Roff, 2010), ubicando ocho puntos en las terminaciones de las venas, uno en la intersección de las venas tres y cuatro, y uno en la articulación apical (Fig. 3); la nomenclatura utilizada se basó en la denominación elaborada por Fedorenko (2009). La cuantificación de las protibias se realizó a través de la descripción de su contorno por medio de descriptores de Fourier. Esta metodología es utilizada cuando se tratan formas biológicas complejas en las que la ubicación por puntos homólogos puede ocasionar una pérdida de información morfológica importante (Lestrel, 1997).

Los análisis de puntos homólogos se efectuaron por medio del paquete de software tpsUtil y tpsDigit (Rolhf, 2008); con la primera aplicación se creó un archivo basado en las imágenes digitalizadas, el cual sirvió de entrada para la segunda utilidad; en esta se ubicaron los landmarks. La matriz de coordenadas (X y $\mathrm{Y})$ resultante, se depuró por medio del algoritmo generalizado para Procrusters (Goodall, 1995). El efecto del tamaño fue eliminado

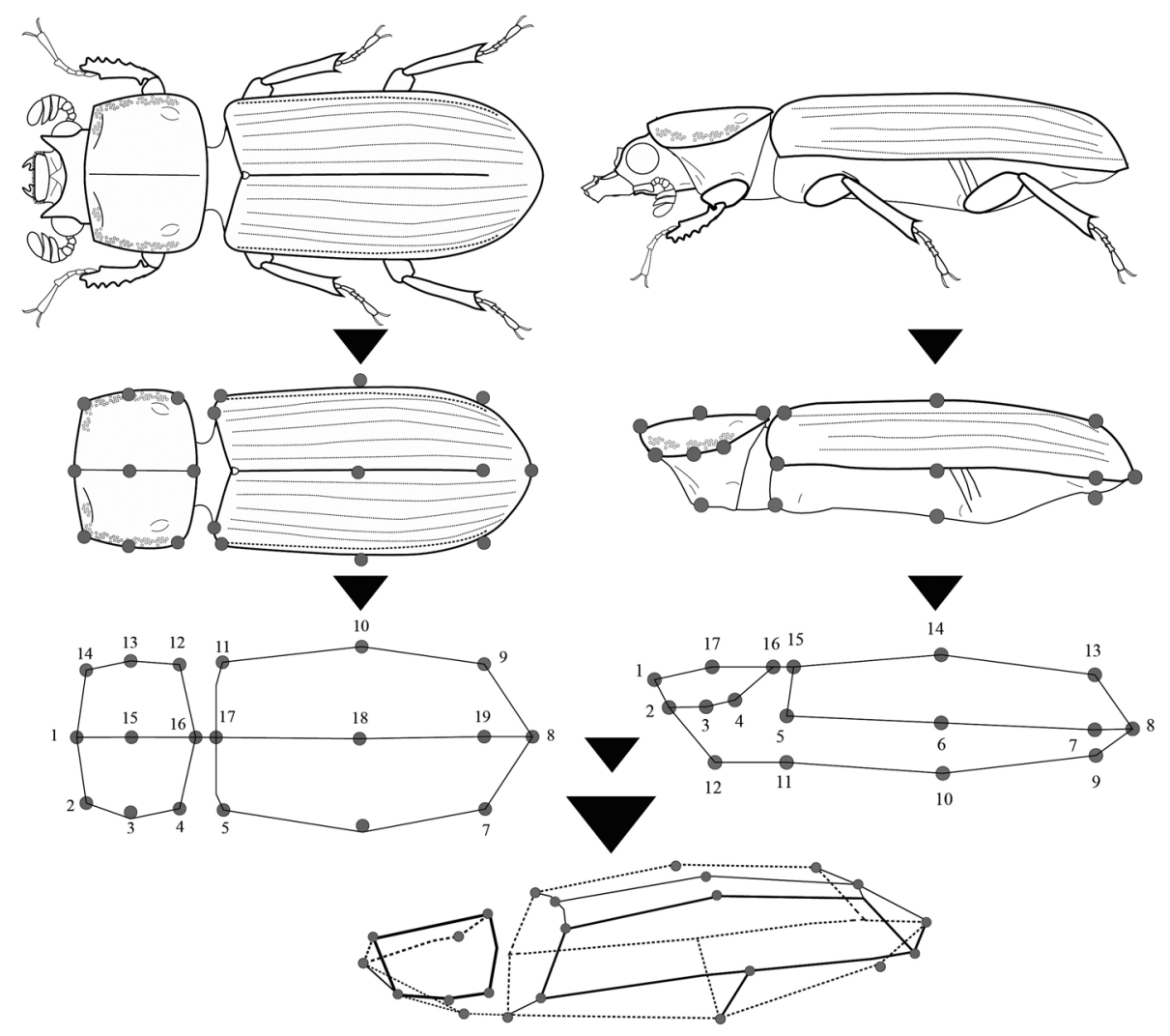

Fig. 2. Mapa de puntos homólogos, dorsales (izquierda) y laterales (derecha), con descriptores (numerados) de la morfología general de pasálidos. Los círculos grises corresponden a puntos de ordenación, los rojos hacen referencia a landmarks de cada plano.

Fig. 2. Landmark map, dorsal (left) and lateral (right) with numeric references. Grey dots refer to ordination points and red dots refer to landmarks. 


\section{Nomenclatura alar}

1 js: esclerotización jugal

2 AP4: anteroposterior cuatro

3 AA3b: anterior anal tres $b$

4 AAP: anterior anal posterior

5 AAl +2: fusión anterioranal uno y dos

MP3+4: fusión medialposterior tres y cuatro

RMP: radiomedial posterior

SV2: vena secundaria dos

aj: articulación apical

10 I-2aS: separación de zonas anteriores uno y dos

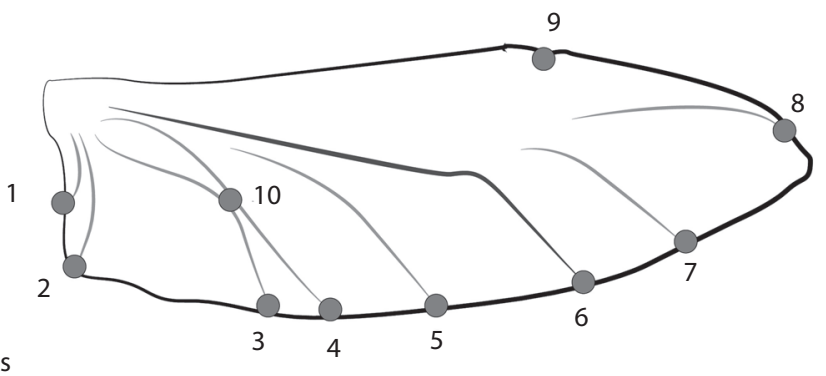

Fig. 3. Puntos homólogos y su nomenclatura (según Fedorenko 2009), empleados como descriptores morfométricos en las alas membranosas de pasálidos en un gradiente altitudinal de robledal en el Parque Natural Municipal Robledales de Tipacoque, PNMRT (Cordillera Oriental, Colombia).

Fig. 3. Landmarks location and names of bess beetles hind wing venation (based on Fedorenko) of an oak forest elevation gradient in Parque Natural Municipal Robledales de Tipacoque PNMRT (Cordillera Oriental, Colombia).

realizando la división de coordenadas sobre el centroide, no obstante esto no excluye un potencial efecto de la alometría (Bookstein, 1991). Los algoritmos utilizados para la transformación de datos brutos junto con los cálculos efectuados para los análisis de ordenación se realizaron por medio del software PAST $v$. 2.17 (Hammer, 2013).

El análisis de series de Fourier, para contornos de protibias, se realizó utilizando 25 harmónicas para lograr una reconstrucción altamente detallada (Rohlf \& Archie, 1984). Cada serie se compone por un número dado de formaciones elípticas, o harmónicas (en este caso 25); por cada cambio en estas figuras, se produce un ajuste gradual direccionado hacia el contorno de la estructura a analizar (Lestrel, 1997), en este caso las protibias. Este proceso se efectuó por medio del software SHAPE 1.3 (Iwata \& Ukai, 2002), específicamente las utilidades ChainCoder (CC) y CHC2NEF (CN); la primera $(\mathrm{CC})$ generó códigos de cadena con respecto a la ubicación de los pixeles asociados al contorno de las estructuras digitalizadas, posteriormente, dicho código fue traducido por $\mathrm{CN}$ a descriptores de series de Fourier.

Con el fin de medir la consistencia de los datos y su independencia frente a los efectos de muestreo, se efectuaron análisis de varianza no paramétricos ANOVA (Kruskal-Wallis) para las variables morfométricas lineales $(\mathrm{L}=$ longitud; $\mathrm{H}=$ grosor corporal; $\mathrm{W}=$ ancho de los élitros y $\mathrm{BW}=$ peso fresco) a nivel altitudinal y gremial. A su vez, se efectuaron regresiones lineales simples entre la longitud del cuerpo (L) con respecto a $\mathrm{H} / \mathrm{W}, \mathrm{L} / \mathrm{W}$ y BW. También se establecieron correlaciones entre peso (BW), longitud (L), amplitud elitral (HW) y longitud elitral (LW) con respecto a la altitud. Todos los análisis se desarrollaron por medio del software IBM SPSS Statistics 20 y se interpretaron gráficamente por medio del software SigmaPlot $v$. 11 (Systat Software, Inc).

Gremios bajo el gradiente: Con el propósito de identificar los cambios en el plan corporal y las alas bajo condiciones del gradiente se utilizó la matriz de Proclusters con un análisis de componentes principales (PCAP); este procedimiento generó estimativos de clasificación con un mayor grado de interpretación. Para estudiar la variación morfológica se aplicó la metodología de deformaciones de cuadrícula, (TPS), en donde se observan los cambios de expansión y contracción de landmarks con respecto a una ubicación promedio (Dryden \& 
Mardia, 1998); la cuadrícula cambia su forma conforme a lo lejano o cercano que se encuentre un determinado punto de su localización promedio dada por los componentes principales (PCs). Para dicha representación se utilizó la herramienta de análisis de componentes principales TPS en dos dimensiones (PCA TPS 2D) del software PAST 2.17; para observar los cambios correspondientes a los valores negativos y positivos de cada PC se usaron factores de expansión, computados por el factor Jacobiano de cada deformación, estos se explicaron a través de variaciones cromáticas en la cuadrícula (matiz azul para contracción, matiz rojo para expansión) (Hammer, 2013).

Por su parte, en el análisis de componentes principales correspondiente a los cambios en los contornos de protibias de pasálidos se utilizó la matriz de harmónicas proveniente de las series de Fourier aplicadas. Estos datos se procesaron a través de la utilidad PrinComp del software SHAPE 1.3 (Iwata \& Ukai, 2002). La interpretación gráfica de los PCA de todos los análisis se realizaron por medio del software SigmaPlot v. 11 (Systat Software, Inc).

\section{RESULTADOS}

Comunidades de pasálidos: Se recolectaron 580 individuos adultos de 198 grupos familiares, pertenecientes a tres gremios $(H=7.74$; $\mathrm{gl}=3 ; \mathrm{P}=0.052)$ (Cuadro 1). La distribución altitudinal de los gremios estuvo relacionada con la riqueza de especies y la abundancia de los grupos familiares; en este sentido, se observó una disminución en las variables mencionadas con el aumento en la elevación. La riqueza gremial más alta se observó a los 2500 y a los $3000 \mathrm{~m}$., en estos puntos se recolectaron grupos familiares en todas las microzonas lo que significa la presencia de todos los gremios en este rango (Cuadro 1).

Los pasálidos alboduramícolas predominaron en el gradiente con respecto a los restantes gremios. Por el contrario, la tendencia subcortícola presentó la menor representación en los puntos altitudinales, de estos sólo se recolectaron grupos familiares a los $2500 \mathrm{msnm}$. En el punto altitudinal mayor $(3300 \mathrm{~m})$ se observó una marcada disminución de gremios. En el robledal ubicado a los $2500 \mathrm{~m}$ se observaron dos colonias de Passalus curtus en la interfaz bosque-suelo. Las categorías de descomposición intermedia en troncos albergaron la mayor variedad de especies y de gremios, mientras que etapas iniciales de descomposición en los troncos se recolectaron pasálidos subcortícolas tanto estrictos (Passalus sp.1) como ocasionales (Passalus irregularis). En el interior de los troncos en avanzada descomposición solo se recolectaron organismos asociados al duramen (Popilius gibbosus).

\section{CUADRO 1}

Número de grupos familiares totales pertenecientes a cinco especies y tres gremios en un gradiente altitudinal de robledal en el Parque Natural Municipal Robledales de Tipacoque, PNMRT (Cordillera Oriental, Colombia)

TABLE 1

Total number of family groups of five species and three guilds on an oak forest under elevation gradient in Parque Natural Municipal Robledales de Tipacoque, PNMRT (Cordillera Oriental, Colombia)

\begin{tabular}{|c|c|c|c|c|}
\hline \multirow[b]{2}{*}{ Alboduramícolas } & \multicolumn{4}{|c|}{ Grupos familiares por microzona } \\
\hline & 2500 & 2700 & 3000 & 3300 \\
\hline Passalus (Petrejus) curtus & 11 & 41 & 11 & 16 \\
\hline Passalus (Pertinax) quyefutynsuca & 8 & 15 & 6 & 0 \\
\hline Poplilus gibbosus & 4 & 12 & 1 & 0 \\
\hline \multicolumn{5}{|l|}{ Generalista } \\
\hline Passalus (Pertinax) irregularis & 5 & 31 & 14 & 13 \\
\hline \multicolumn{5}{|l|}{ Subcortícola } \\
\hline Passalus (Pertinax) sp.1 & 10 & 1 & 0 & 0 \\
\hline
\end{tabular}


CUADRO 2

Diferencias entre las mediciones morfológicas lineales de pasálidos, con relación a la altitud y a los gremios

TABLE 2

Lineal morphological measures change of bess beetles with elevation and guild reference

\begin{tabular}{lcccccc}
\multicolumn{1}{r}{} & Altitudinal & \multicolumn{3}{c}{ Entre gremios } \\
Medida & Df & F & P & df & F & P \\
L & 3 & 0.22 & 0.88 & 2 & 40.1 & $0.01^{*}$ \\
BW & 3 & 0.16 & 0.92 & 2 & 28.6 & $0.01^{* *}$ \\
HW & 3 & 0.41 & 0.74 & 2 & 5.9 & $0.01^{+}$ \\
LW & 3 & 0.04 & 0.98 & 2 & 12.1 & $0.02^{+}$ \\
\hline
\end{tabular}

*Diferencias significativas entre todos los gremios.

**Diferencias significativas mayores entre alboduramícolas y generalistas.

+ Diferencias significativas mayores entre subcortícolas y alboduramícolas.

$\mathrm{L}=$ longitud total. $\mathrm{BW}=$ peso fresco. $\mathrm{HW}=$ espesor de élitros. $\mathrm{LW}=$ longitud de élitros

$\mathrm{L}=$ total length. $\mathrm{BW}$ : fresh weight. $\mathrm{HW}=$ elitral width. $\mathrm{LW}=$ elitral length.

Morfometría lineal y gremios: Las variables morfométricas lineales estudiadas mostraron una independencia con respecto a los gremios, pero no así con respecto al gradiente (Cuadro 2). No obstante, el peso fresco (BW), la longitud total del cuerpo (L) y la proporción espesor-longitud del cuerpo $(\mathrm{H} / \mathrm{L}$,) en cada uno de los gremios, mostraron una tendencia a incrementarse con respecto a la altitud. Por el contrario, la proporción ancho de élitros-longitud del cuerpo (W/L) presentó el patrón opuesto, es decir disminuyó con respecto a la altitud. Se observó una correlación positiva de $\mathrm{L}$ con respecto a $\mathrm{BW}(\mathrm{R}=0.69 ; \mathrm{df}=1 ; \mathrm{P}=0.04)$ y $\mathrm{WL}(\mathrm{R}=0.81 ; \mathrm{df}=1 ; \mathrm{P}=0.01)$. Los generalistas y subcortícolas se asociaron a especies de pequeña talla $(\leq 2.5 \mathrm{~cm})$ y menor biomasa $(\leq$ $0.5 \mathrm{~g}$ ); los pasálidos alboduramícolas oscilaron entre organismos de talla media $(\approx 2.8 \mathrm{~cm})$ y mayor biomasa $(\approx 1.3 \mathrm{~g})$, y entre organismos de mayor longitud $(3.3 \mathrm{~cm})$ y biomasa media $(0.9$ g) (Fig. 4A). En este sentido, la correlación de estas dos variables se estableció independientemente del gradiente altitudinal. Por otra parte, la correlación entre la longitud corporal (L) y la relación $\mathrm{L} / \mathrm{HW}$ evidenció asociaciones a nivel altitudinal y gremial. En el rango de los $2700 \mathrm{~m}$ hasta los $3300 \mathrm{~m}$ se agruparon pasálidos de dos especies alboduramícolas (Popilius gibbosus y Passalus quyefutynsuca), acompañadas de una especie generalista (Passalus irregularis encontrada a los $3300 \mathrm{~m}$ ) (Fig. 4B). La especie Passalus curtus mostró los mayores valores en $\mathrm{L}$ y L/HW, sin embargo, los organismos recolectados a los $3300 \mathrm{~m}$ se agruparon con las restantes especies de preferencia alboduramícola y con P. irregularis (hallada a $3300 \mathrm{~m}$, que corresponde a la máxima altitud).

No se observó una relación entre L y HW $(\mathrm{R}=-0.72 ; \mathrm{df}=1 ; \mathrm{P}=0.002$. En este caso, se agrupan pasálidos con tendencias alboduramícolas provenientes de todo el gradiente junto a generalistas presentes a $3300 \mathrm{~m}$., los cuales comparten valores medios de la proporción HW y de L (Fig. 4C). Los generalistas (2 500 $3000 \mathrm{~m}$ ) y subcortícolas se asociaron a valores altos de HW y bajos de L, los alboduramícolas restantes se ajustaron a una relación inversa con respecto a los pasálidos subcortícolas.

Morfometría geométrica y gremios: En los análisis de ordenación (PCA) correspondientes al análisis de series de Fourier para protibias y landmarks el cambio en la forma de contornos y la ubicación de puntos homólogos se explica en tres componentes principales (PCs). La variación entre los landmarks situados en las alas anteriores se explicó en dos PCs (Cuadro 3). En el análisis de ordenación basado en los puntos homólogos seleccionados en 

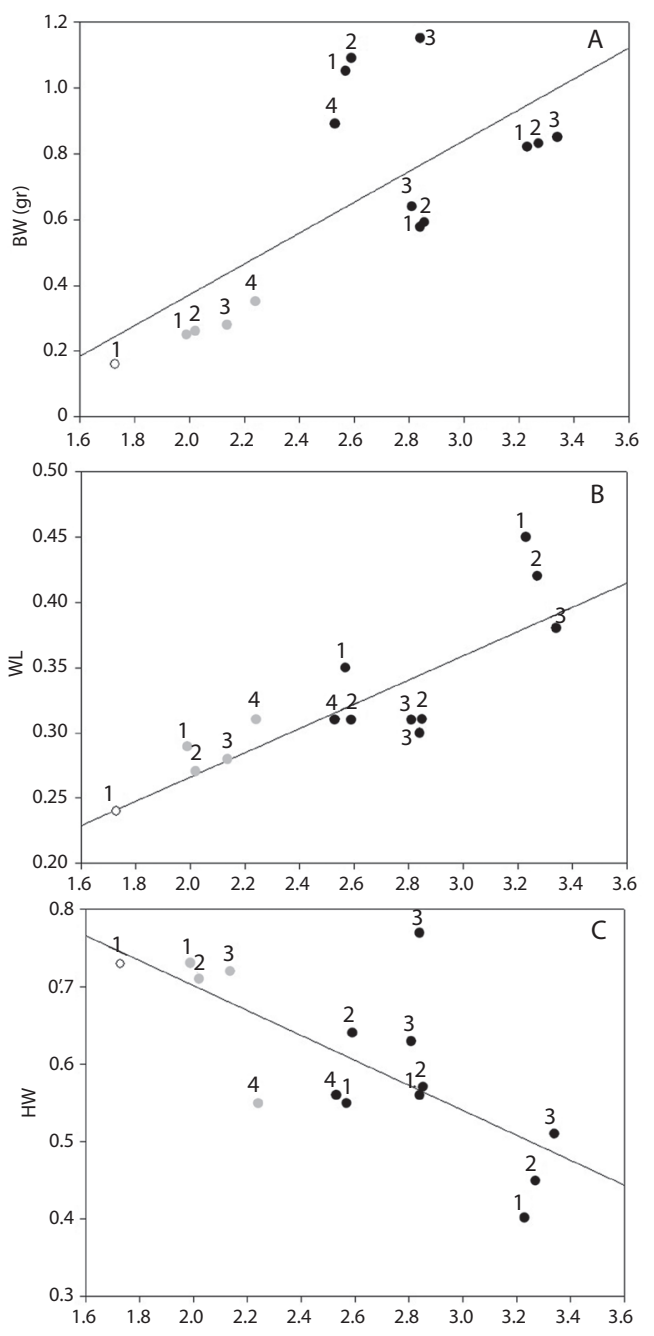

alas de pasálidos, la variación total se explicó a través de dos componentes: el componente principal uno representó un gradiente de expansión y contracción de los puntos ubicados en la zona anterior, media y posterior del ala, junto a la articulación apical (Fig. 5). El segundo componente albergó la expansión y contracción de los puntos tres y cuatro (zona anterior del ala).

Se observaron dos claras agrupaciones a partir de dichos componentes, la primera se compone Passalus curtus, cuyos puntos ubicados en las venas anteriores y medias se expandieron y los ubicados en la zona posterior junto a la articulación apical se contrajeron con respecto a la ubicación promedio de puntos
Fig. 4. Correlaciones entre medidas morfológicas lineales de pasálidos en un gradiente altitudinal de robledal en el Parque Natural Municipal Robledales de Tipacoque, PNMRT (Cordillera Oriental, Colombia). (a) BW (peso fresco) vs L (longitud corporal), (b) LW (longitud elitral) vs L (longitud corporal), (c) HW (espesor élitros) vs L (longitud corporal). Los círculos negros hacen referencia a organismos alboduramícolas, los grises a generalistas y los círculos vacíos a subcortícolas. Los números corresponden a los puntos altitudinales 1 (2500 m.), 2 (2700 m.), 3 (3000 m.) y 4 (3300 m.).

Fig. 4. Lineal morphological measures correlation on bess beetles from an oak forest elevation gradient in Parque Natural Municipal Robledales de Tipacoque, PNMRT. (A) BW (fresh weight) vs L (total length), (B) LW (elitral length) vs L (total length), and (C) HW (elitral width) vs $\mathrm{L}$ total length. Black dots refer to sapwood-heartwood guild, grey dots refer to generalist guild and empty dots to underbark guild. Numbers refer to elevation points 1 (2 500 m.), 2 (2 700 m.), 3 (3000 m.) y 4 (3300 m.).

(hemibraquipterismo) (Fig. 5). Los pasálidos restantes se agruparon hacia el área de valores negativos del componente uno, donde los landmarks situados en las venas de la zona anterior se contrajeron, los de la zona media permanecieron constantes y los de la zona posterior se expandieron.

La descripción de la forma general del cuerpo de Passalidae, basada en puntos homólogos situados dorsal y lateralmente se resumió en tres componentes principales (Fig. 6). El primer componente albergó la variación gradual entre la contracción (PC1-) y expansión (PC1+) de landmarks ubicados desde el pronoto y el húmero hasta la mitad del área elitral (Fig. 6A). Por su parte el PC2 reunió el cambio

\section{CUADRO 3}

Marcadores morfogeométricos considerados en pasálidos, según PCs (análisis de componentes principales)

TABLE 3

Morphogeometric markers shifts based on PCs (principal components analysis)

\begin{tabular}{lcccc} 
& PC1 & PC2 & PC3 & Total (\%) \\
Protibias & 40.89 & 18.35 & 14.3 & 73.54 \\
Dorsal & 35.88 & 22.93 & 12.28 & 71.09 \\
Lateral & 33.16 & 16.79 & 14.5 & 64.45 \\
Alas & 61.42 & 14.076 & - & 75.496 \\
\hline
\end{tabular}




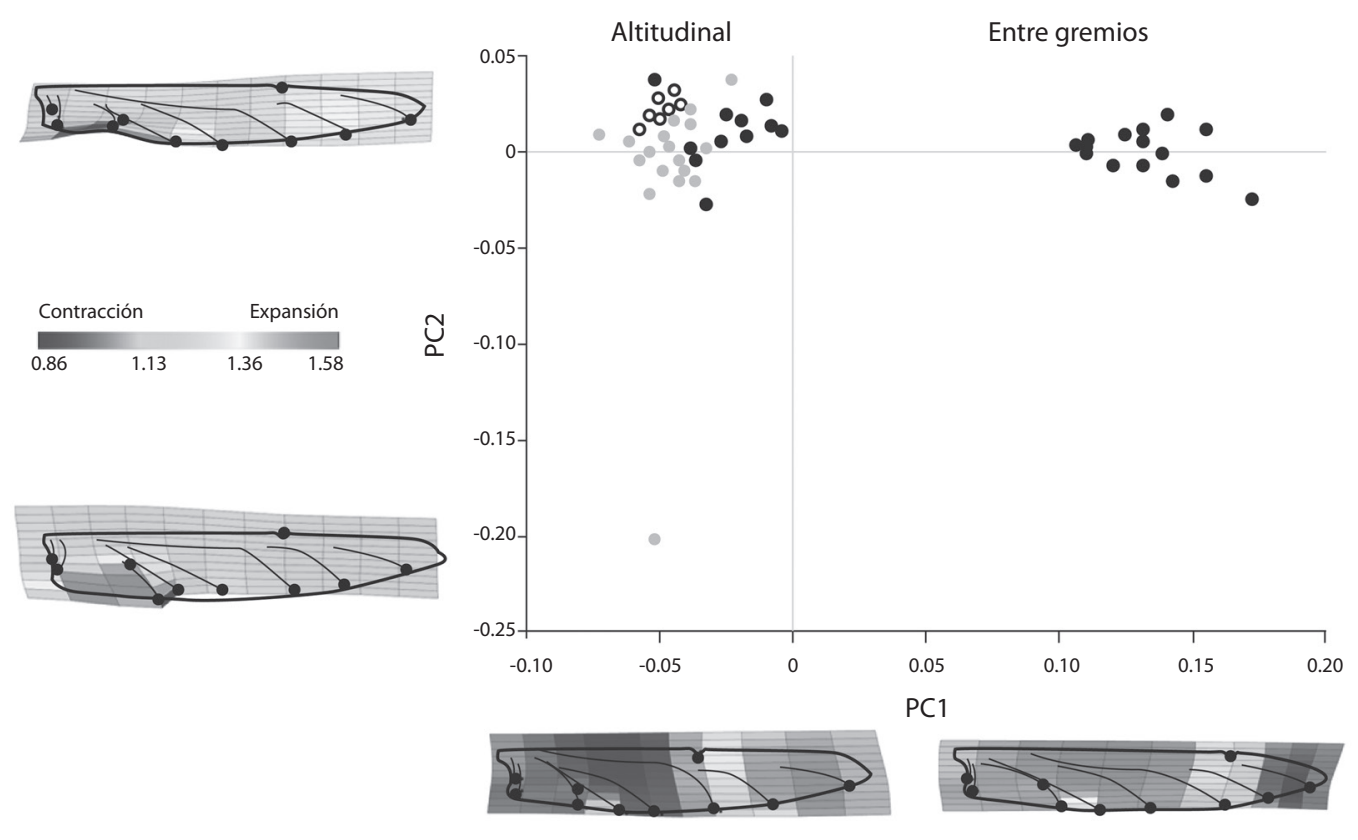

Fig. 5. Análisis de componentes principales para landmarks ubicados en alas anteriores de pasálidos en un gradiente altitudinal de robledal en el Parque Natural Municipal Robledales de Tipacoque, PNMRT. Los círculos negros hacen referencia a organismos alboduramícolas, los grises a generalistas y los círculos vacíos a subcortícolas.

Fig. 5. Principal components analysis of hind wing venation landmarks of bess beetles from an oak forest elevation gradient in Parque Natural Municipal Robledales de Tipacoque, PNMRT. Black dots refer to sapwood-heartwood guild, grey dots refer to generalist guild and empty dots to underbark guild.

en la localización de puntos homólogos extremos; en este se reconocieron formas corporales ensanchadas hacia el centro y con extremos reducidos, y viceversa (Fig. 6B). Por último, se observaron patrones morfológicos caracterizados por cuerpos marcadamente expandidos o reducidos, excepto por los extremos superiores (pronoto-húmero) e inferiores (margen externo de élitros) (PC3). Los alboduramícolas se agruparon bajo patrones corporales convexos con una notable expansión en la zona del pronoto y del abdomen; en el caso puntual de Passalus curtus se reconoce una mayor influencia de la expansión de los puntos finales situados en la margen externa de los élitros (Fig. 6A y Fig. 6B). Los generalistas, representados por Passalus irregularis, se ubicaron cerca a la descripción promedio de la forma, con propensión a cuerpos de menor espesor en los extremos superiores e inferiores (Fig. 6A). Por último, la morfología general de los pasálidos subcortícolas se caracterizó por cuerpos estrechos dorsalmente desde el pronoto hasta tres cuartos de los élitros, y lateralmente desde el proesternón hasta el metasternón (Fig. 6A y Fig. 6B).

Por su parte, el análisis de ordenación basado en series de Fourier a partir de los contornos de protibias, arrojó claras agrupaciones gremiales más no altitudinales. La transformación de los contornos se resumió en tres componentes, el primero (PC1) comprendió la ubicación de denticiones de la margen exterior de la estructura y la agudeza de dichas proyecciones; el PC2 albergó el número de denticiones y el ensanchamiento de la zona apical y por último el PC3 reunió el cambio del ancho total de la estructura y la separación entre denticiones (Fig. 7). A partir de dichos componentes se reconocen agrupaciones gremiales claras. Los subcortícolas se reúnen en contornos caracterizados por un rango de tres a cuatro denticiones 


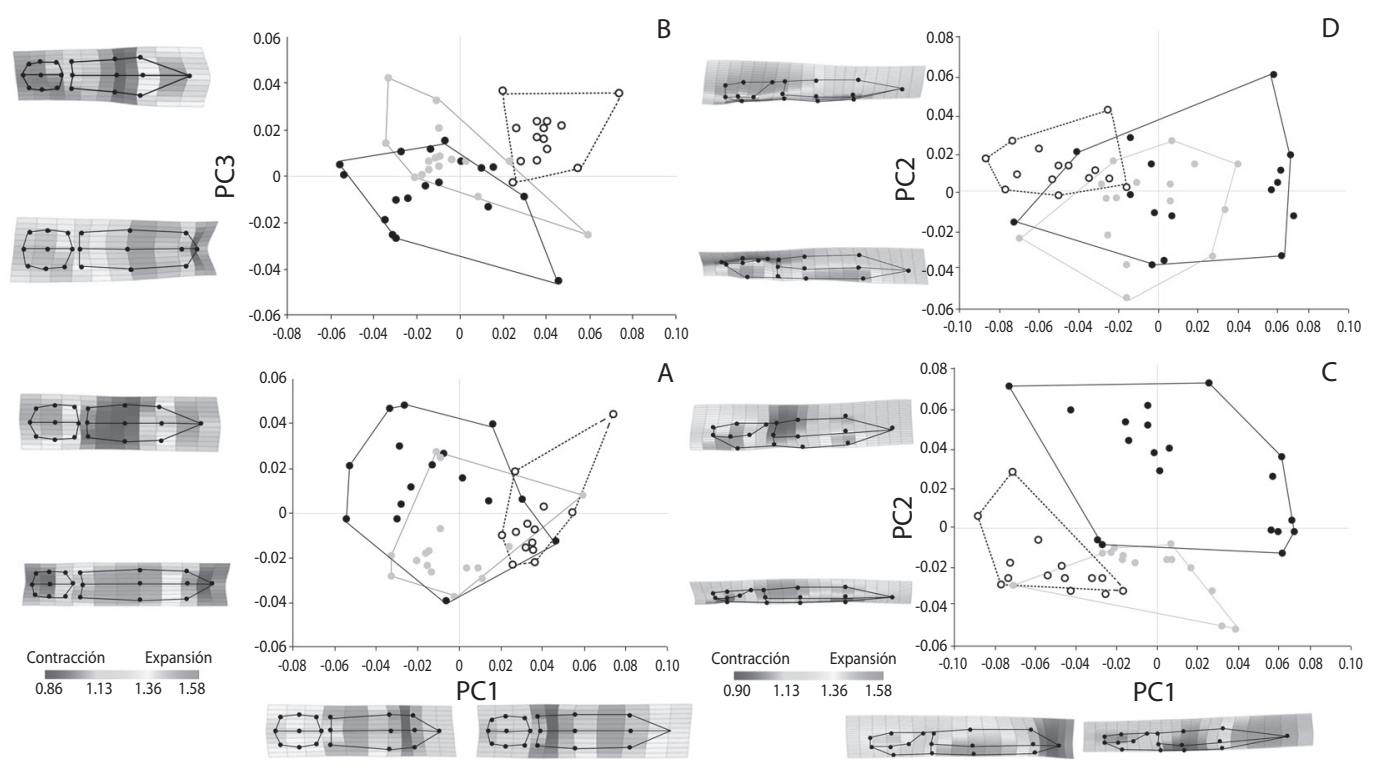

Fig. 6. Análisis de componentes principales para landmarks, ubicados dorsalmente (A) PC1 VS PC2; (B) PC1 vs. PC3; y lateralmente (C) PC1 VS PC2; y (D) PC1 vs. PC3. Los círculos negros hacen referencia a organismos alboduramícolas, los grises a generalistas y los círculos vacíos a subcortícolas.

Fig. 6. Principal components analysis landmarks, dorsally located (A) PC1 VS PC2; (B) PC1 vs. PC3; and sideways (C) PC1 VS PC2; y (D) PC1 vs. PC3. Black dots refer to sapwood-heartwood guild, grey dots refer to generalist guild and empty dots to underbark guild.

agudas, ubicadas en la zona apical del margen externo de la protibia, que es a su vez una zona con un ensanchamiento con respecto a la base. El conjunto de pasálidos generalistas mostró un contorno variable en ancho general, en número y agudeza de denticiones; su forma se distinguió de los otros gremios por un mayor espacio entre dichas proyecciones. En cuanto al gremio de los alboduramícolas, se ajustaron a contornos con un ancho uniforme en toda la estructura, con un número de denticiones agudas desde seis hasta ocho y menor separación entre ellas (Fig. 7).

\section{DISCUSIÓN}

Las variables morfométricas básicas medidas en este estudio, referentes al tamaño corporal, mostraron un patrón positivo con respecto a la altitud. Específicamente, la longitud y biomasa aumentaron en toda la comunidad al incrementar la altitud. En insectos, el incremento en el tamaño se explica en general por una relación negativa entre la temperatura óptima de desarrollo y el tamaño en ambientes donde el recurso no esta limitado (Smith et al., 2000; Chown \& Klok, 2003). A su vez, si este cambio es gradual, la condición univoltina reviste un carácter importante.

El estudio de Lobo y Castillo (1997) muestra una correlación con tendencia positiva entre HW y L, con una marcada diferencia entre pasálidos de ambas tribus de la subfamilia Passalinae: Passalini y Proculini, estos últimos presentan una mayor proporción en los valores de HW. En la comunidad de Passalidae, bajo el gradiente altitudinal del robledal, no se observó dicha segregación de tribus, pero sí se dio, en cambio, un patrón de correlación negativa con agregación de especies de puntos de elevación específicos.

Para las variables mencionadas y en las especies Passalus irregularis (generalista), Passalus curtus, Popilius gibbosus y Passalus quyefutynsuca (alboduramícolas) se observó una correlación positiva, contrario a lo 

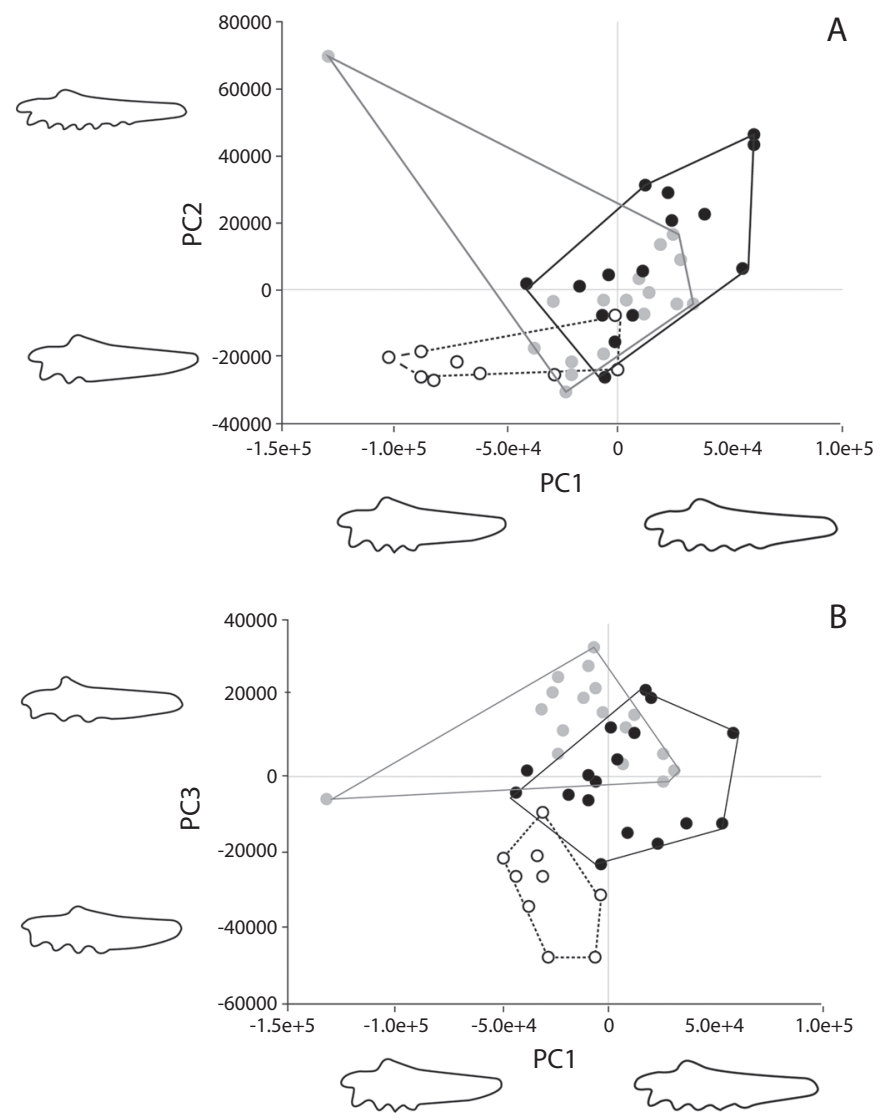

Fig. 7. Análisis de componentes principales para series de Fourier, de contornos de protibias de escarabajos pasálidos (A) PC1 vs. PC2; y (B) PC1 vs PC3. Los círculos negros hacen referencia a organismos alboduramícolas, los grises a generalistas y los círculos vacíos a subcortícolas.

Fig. 7. Principal components analysis Fourier series of bess beetle protibiae contours (A) PC1 vs. PC2; y (B) PC1 vs PC3. Black dots refer to sapwood-heartwood guild, grey dots refer to generalist guild and empty dots to underbark guild.

registrado por Johki y Kon (1987) y Lobo y Castillo (1997). Las diferencias entre el grosor y las medidas referentes a la forma corporal, evidentes en los grupos taxonómicos menos basales filogenéticamente de Passalidae (Reyes-Castillo \& Halffter, 1984), probablemente obedezcan a cambios altitudinales; estos se consideran como los factores históricos moldeadores de la estructura de la comunidad en los pasálidos de la alta montaña andina (Amat \& Reyes-Castillo, 2002). Si bien la diversidad morfológica de Passalidae se considera homogénea, basada en patrones morfométricos similares, se debe tener en cuenta que el carácter de las medidas lineales que han determinado la modelación del groundplan específico pueden ser influenciadas por factores históricos locales; a su vez, dicha variación podría ser debida a respuestas ecológicas relacionadas con la incorporación de energía a partir del recurso alimento (Horgan, 2005; Chown \& Gaston 2010).

En los Passalidae, las alas membranosas cumplen funciones de movimiento y de comunicación. El desarrollo de estas estructuras y su polimorfismo, han sido referentes importantes de la evolución de la familia (Reyes-Castillo \& Jarman, 1983; Boucher, 2005). En algunos casos, principalmente en pasálidos alboduramícolas, las especies han perdido la capacidad 
de volar y presentan alas atrofiadas parcial (hemibraquipterismo) o totalmente (braquipterismo), con la intervención de un órgano estridulatorio (Reyes-Castillo, 1970; Schuster \& Schuster, 1971; Boucher, 2005). La configuración de puntos homólogos situados en las venas alares de pasálidos del presente estudio, se ajustó a organismos macrópteros (capacidad total de vuelo) y una especie hemibraquíptera (Passalus curtus). En esta especie, se observó un desplazamiento de landmarks ubicados en la zona radial posterior del ala debido a la reducción en tamaño de dicha área. Es posible que la modificación de esta estructura aumente los rangos de comunicación al interior del tronco (mayor área de contacto de microespinas alares), permitiéndole a Passalus curtus un aprovechamiento selectivo de albura y duramen para reproducción y alimentación. La variación morfológica alar de Passalus curtus, pudo darse en respuesta a presiones selectivas específicas, posiblemente explicadas por el aislamiento altitudinal o una insularización secundaria (Boucher, 2005).

Dentro de los pasálidos, con la configuración de puntos homólogos acorde a la condición de alas desarrolladas, se observaron agrupaciones a nivel gremial. Las diferencias se basaron en pequeñas modificaciones en las venas ubicadas en la zona anterior de las alas; los subcortícolas y los generalistas exhibieron un mayor factor de expansión en el área específica. A nivel funcional, es posible que el presentar alas desarrolladas haya influido en la capacidad de explotación de la subcorteza, microzona que se caracteriza por su carácter efímero (Reyes-Castillo \& Halffter, 1984). Esta característica permite una mayor distribución y búsqueda de troncos (Ranius, 2006; Jackson, Baum, Robert, \& Cronin, 2009) (subcortícolas a $2500 \mathrm{~m}$ ), y a su vez un movimiento eficiente a lo largo del gradiente (generalistas 2500-3300 m).

El análisis morfogeométrico mostró tendencias relacionadas por su patrón corporal, con respecto a una explotación similar del recurso. En detalle, organismos subcortícolas se caracterizan por sus formas aplanadas dorso-lateralmente con la mayor expansión alojada en el pronoto, húmero, élitros, prosterno y abdomen. Por su parte, los landmarks ubicados en el cuerpo de los pasálidos alboduramícolas se caracterizan por sus formas cóncavas con la mayor expansión albergada en el pronoto, como ha sido documentado por varios autores (Reyes-Castillo, 1970, Schuster, 1978; Reyes-Castillo \& Halffter, 1984; Lobo \& Castillo, 1997; Castillo \& Reyes-Castillo, 2003). Al respecto, los datos de este estudio presentan una mayor definición morfológica en ambos gremios. Adicionalmente, se destaca la heterogeneidad de formas dentro de Passalus irregularis, única especie con cierta plasticidad por sus tendencias mixtas en los patrones de alimentación y ocupación del espacio.

A pesar de exhibir una forma similar a Passalus sp.1, la configuración morfométrica de Passalus irregularis comparte las características de ambos gremios especialistas. Es posible que este patrón corporal presente ventajas al explotar las diversas microzonas del tronco en descomposición, lo suficientemente cóncavo para desenvolverse en la albura y el duramen, y aplanado para la subcorteza. Debido a esto, es factible que el tiempo de permanencia y reproducción de esta especie generalista en determinada microzona, sea mediada por la presencia de otros pasálidos con un mayor grado de especialización.

La madera en descomposición se caracteriza por tener una menor consistencia sólida respecto a un tronco de un árbol vivo, a pesar de esto, el acceso al interior de los troncos caídos representa un proceso complejo para los Passalidae. En respuesta a esto, los escarabajos pasálidos exhiben mandíbulas altamente esclerotizadas con una fuerza muscular suficiente para remover la corteza y la madera del interior del tronco. Estas adaptaciones han permitido la elaboración de túneles y galerías dentro de los troncos, y a su vez, han posibilitado la alimentación de los adultos, quienes fragmentan la madera y extraen trozos pequeños; estos fragmentos, adicionalmente, son consumidos por las larvas. Se ha descrito la acción conjunta de patas delanteras y de movimientos oscilatorios 
de la cabeza (cuernos) en el proceso de fragmentación de la madera. Las protibias constituyen estructuras de primer orden que influyen en dicha acción (Reyes-Castillo \& Jarman, 1981; 1985). Los análisis de series de Fourier arrojaron claras agrupaciones de los contornos de estas estructuras, que obedecieron a la composición de gremios. Las protibias de los pasálidos subcortícolas exhibieron contornos con pocas denticiones agudas, ubicadas en la parte apical, con un grosor mayor en dicha área con respecto al total de la tibia. Los alboduramícolas, por su parte, exhibieron tibias con un ancho uniforme, con múltiples denticiones agudas espaciadas entre sí; la especie generalista presentó denticiones variables en número, con más espacio entre sí y con un ensanchamiento gradual hacia su ápice. Es factible que la forma diferencial de este complejo morfológico este relacionada con un mejor manejo del recurso en las microzonas específicas. Las pocas denticiones en el área más ancha de las protibias de escarabajos subcortícolas pueden actuar ejerciendo presiones opuestas para desplazar la corteza y generar un espacio en la interfaz con el tronco. Por el contrario, tibias con ancho fijo y mayor cantidad de denticiones agudas, pueden actuar no solo como instrumentos de agarre sino de fragmentación.

\section{AGRADECIMIENTOS}

Agradecemos a la alcaldía del municipio de Tipacoque, Boyacá, Colombia por la disposición y atención prestada durante la fase de campo. A Karen Salazar y Nini Johana Beltrán por su vital ayuda en campo. A Pedro ReyesCastillo por el acompañamiento y asesoría a esta investigación y a María Luisa Castillo por sus valiosos aportes al manuscrito.

\section{RESUMEN}

Los escarabajos pasálidos son componentes importantes de los bosques tropicales debido a que facilitan el reciclaje de nutrientes propios de madera en descomposición, ya sea por acción directa (consumo) o indirecta (facilitando la acción de otros organismos). En la actualidad los estudios ecológicos dentro del grupo son escasos y se han centrado en respuestas a cambios ambientales relacionados con su recurso alimenticio. En el presente estudio se caracterizó la composición gremial de escarabajos pasálidos, distribuida en un gradiente altitudinal, de acuerdo a sus características de aprovechamiento del recurso. Se cuantificaron aspectos morfoecológicos de importancia adaptativa para la explotación diferencial del recurso (medidas corporales, puntos homólogos en cuerpo y alas y series de Fourier en protibias). Se reconocieron cinco especies, 198 grupos familiares y tres gremios: el subcortícola, el alboduramícola y el generalista. La riqueza de la comunidad disminuyó conforme aumentó la altitud. Los cambios entre las variables morfométricas lineales se vieron influenciadas por la altitud; por su parte, los datos morfogeométricos se comportaron independientes del gradiente. El análisis de marcadores morfológicos (morfogeométrico) arrojó información relevante para la delimitación de los gremios ya establecidos en esta familia. Dentro de estos, la forma general del cuerpo junto a las tibias anteriores, aportaron la mayor información de agrupación gremial. La cuantificación de dichos marcadores evidenció la relación entre los tipos de aprovechamiento del recurso, ya que al ser estructuras importantes en el movimiento y consumo de la madera en microzonas particulares, permite plantear roles funcionales puntuales. Reconocer estas variables en interacción con aquellas ecológicas brinda herramientas para el entendimiento de la riqueza y los aportes funcionales de los escarabajos pasálidos al ecosistema.

Palabras clave: ecología funcional, gremio, morfometría geométrica, Passalidae.

\section{REFERENCIAS}

Amat, G. D. \& Reyes-Castillo, P. (2002). Los Coleoptera Passalidae de Colombia. En C. Costa, S. Vanin, \& J. Lobo (Eds.). (2002). Pribes 2002: Proyecto Iberoamericano de Biogeografía y Entomología Sistemática (Vol. 2, pp. 139-151). Monografías Tercer Milenio. SEA, España.

Biewener, A. A. (2003). Animal Locomotion. Oxford, UK: Oxford University Press.

Blondel, J. (2003). Guilds or functional groups: does it matter? Oikos, 100, 223-231.

Bookstein, F. L. (1991). Morphometric, Tools for Landmark Data: Geometry and Biology. Cambridge University Press.

Boucher, S. (1991). Les Passalides de l' archipel du Vanuatu. Remarques faunistiques et biogéographiques; comparaison avec la Nouvelle-Calédonie (Coleoptera: Passalidae). Annales de la Société Entomologique de France (n.s), 27(1), 361-374. 
Boucher, S. (2005). Évolution et phylogénie des Coleóptéres Passalidae (Scarabaeoidea). International Journal of Entomology, 41(3-4), 239-604.

Castillo, M. L. \& Morón, M. A. (1992). Observaciones sobre la degradación de madera por algunas especies de pasálidos (Coleoptera: Lamellicornia). Folia Entomológica Mexicana, 84, 35-44.

Castillo, M. J. L. \& Reyes-Castillo, P. (2003). Los Passalidae: coleópteros degradadores de troncos de árboles muertos. En J. Álvarez-Sánchez \& E. Naranjo García (Eds.). Ecología del Suelo en la selva tropical húmeda de México (pp. 237-262). México: Instituto de Ecología, A.C., Instituto de Biología y Facultad de Ciencias, UNAM. Xalapa, México.

Chown, S. L., \& Gaston, K. J. (2010). Body size variation in insects: a macroecological perspective. Biological Reviews, 85, 139-169.

Chown, S. L., \& Klok, C. J. (2003). Altitudinal body size clines: latitudinal effects associated with changing seasonality. Ecography, 26, 445-455.

Dryden, I. L., \& Mardia, K. V. (1998). Statistical Shape Analysis. Wiley.

Fedorenko, D. (2009). Evolution of the beetle hind wing, with special reference to folding (Insecta, Coleoptera). Sofia, Bulgaria: Pensoft Publishers.

Goodall, C. R. (1995). Procrustes method in the statistical analysis of shape revisited. In K. V. Mardia, \& C. A. Gill (Eds), Proceedings in Current Issues in a Statistical Analysis of Shape (pp. 18-39). Leeds: Leeds University Press.

Hammer, O. (2013). PAST: PAleontogical STatistics 3.0. Retrieved from http://folk.uio.no/ohammer/past/ index.html.

Hill, R. W., Wyse, G. A., \& Anderson, M. (2008). Animal Physiology. Segunda Edición. Sunderland, MA: Sinauer Associates.

Horgan, F. G. (2005). Effects of deforestation on diversity, biomass and function of dung beetles on the eastern slope of the Peruvian Andes. Forest Ecological Management, 216, 117-133.

Iwata, H., \& Ukai, Y. (2002). SHAPE: A computer program package for quantitative evaluation of biological shapes based on elliptic Fourier descriptors. Journal of Heredity, 93, 384-385.

Jackson, H. B., Baum, K., Robert, T., \& Cronin, J. (2009). Habitat-specific movement and edge-mediated behavior of the saproxylic insect Odontotaenius disjunctus (Coleoptera: Passalidae). Environmental Entomology, 38(5), 1411-1422

Johki, Y. \& Kon, M. (1987). Morpho-ecological analysis on the relationship between hábitat and body shape in adult Passalid beetles (Coleoptera: Passalidae). Memoirs of the Faculty of Science, Kyoto University (Ser. Biol), 12(2), 119-128.
Klingenberg, C. P., Debat, V., \& Roff, D. (2010). Quantitative genetics of shape in cricket wings: developmental integration in a functional structure. Evolution, 64(10), 2935-2951.

Kon, M., \& Johki, A. (1987). A new type of microhabitat, the interface between the log and the ground, observed in the passalid beetle of Borneo Taeniocerus bicanthatus (Coleoptera: Passalidae). Journal of Ethology, 5, 197-198.

Lestrel, P. (1997). Fourier descriptors and their applications in biology. Cambridge: Cambridge University.

Lobo, J. M., \& Castillo, M. L. (1997). The relationship between ecological capacity and morphometry in a neotropical community of Passalidae (Coleoptera). The Coleopterists Bulletin, 51(2), 147-153.

Loreau, M. (1998). Biodiversity and ecosystem functioning: a mechanistic model. Proceedings of the National Academy of Sciences of the America, 95, 5632-5636

Mouzinho, J. R. C., \& Da Fonseca, C. R. (1998). Contribucao ao estudo da passalidofauna (Coleoptera: Lamellicornia: Passalidae) em uma área de terra firme de Amazonia central. Acta Zoológica Mexicana (n.s), 73, 19-44.

Ranius, T. (2006). Measuring the dispersal of saproxylic insects: a key characteristic for their conservation. Population Ecology, 48, 177-188.

Rauum, R. (2006). DVLR.msi (Dorsal-Ventral-Left-Right fitting) user guide. Retrieved from http://pages.nycep. org/nmg/programs.html

Reyes-Castillo, P. (1970). Coleoptera Passalidae: morfología y división en grandes grupos; géneros americanos. Folia Entomológica Mexicana, 20-22, 1-240.

Reyes-Castillo, P. \& Halffter, G. (1984). La estructura social de los Passalidae (Coleoptera: Lamellicornia). Folia Entomológica Mexicana, 61, 49-72.

Reyes-Castillo, P., \& Jarman, M. (1981). Some notes on larval stridulation in neotropical Passalidae (Coleoptera: Lamellicornia). The Coleopterists Bulletin, 34, 263-270.

Reyes-Castillo, P. \& Jarman, M. (1983). Disturbance sounds of adult passalid beetles (Coloptera: Passalidae): Structural and functional aspects. Annals of the Entomological Society of America, 76(1), 6-22.

Reyes-Castillo, P., \& Jarman, M. (1985). Mandibular force of adult and larvae Passalidae in family groups. Acta Zoológica Mexicana (n.s.), 12, 13-22.

Rohlf, F. J., \& Archie, W. J. (1984). A comparison of Fourier methods for the description of wing shape in mosquitoes (Diptera: Culicidae). Systematic Zoology, 33, 302-317. 
Rolhf, F. J. (2008). TPSdigit 2.12, Ecology \& Evolution. Retrieved from www.life.bio.sunysb.edu/morph. 302-317.

Schuster, J. C. (1975). Comparative behavior, acoustical signals, and ecology of the new world Passalidae (Coleoptera) (Ph. D. Thesis). University of Florida, Gainesville.

Schuster, J. C. (1978). Biogeographical and ecological limits of new world Passalidae (Coleoptera). The Colleopterists Bulletin, 32(1), 21-28.

Schuster, J. C. \& Schuster, L. (1971). Un esbozo de señales auditivas y comportamiento de Passalidae (Coleoptera) del nuevo mundo. Revista Peruana de Entomología, 14(2), 249-252.
Schuster, J. C., \& Schuster, L. (1985). Social behavior in passalid beetles (Coleoptera: Passalidae): cooperative brood care. Florida Entomologist, 68(2), 266-272.

Smith, R. J., Hines, A., Richmond, S., Merrick, M., Drew, A., \& Fargo, R. (2000). Altitudinal variation in body size and population density of Nicrophorus investigator (Coleoptera: Silphidae). Environmental Entomology, 29, 290-298.

Van Regenmortel, M. H. V. (2004). Reductionism and complexity in molecular biology. EMBO Reports, 5, 1016-1020.

Villemant, C., Simbolotti, G., \& Kenis, M. (2007). Discrimination of Eubazus (Hymenoptera, Braconidae) sibling species using geometric morphometric analysis of wing venation. Systematic Entomology, 32, 625-634. 
